\title{
1
}

\section{Introduction to Wireless Propagation}

\subsection{Introduction}

This book deals with several topics related to mobile and, in general, wireless propagation channels using a simulation approach. This means that we will be generating synthetic, but realistic, series of relevant propagation parameters as a function of time or traversed distance. It is hoped that this will allow the reader to become acquainted with this topic in a simpler, more intuitive way than by using cumbersome mathematical formulations.

Typically, frequencies in the VHF/UHF bands, and slightly above, are used for wireless applications. A number of propagation mechanisms such as reflections, diffractions, transmissions, etc. dominate. These effects are normally caused by environmental features close to the user terminal or mobile station, MS. In some cases, also the other end of the link, the base station, BS, or network access point will be surrounded by local features affecting the propagation characteristics. Furthermore, in some cases, far-off, large environment elements such as mountains or buildings may also intervene in the link characteristics, causing significant time spreading.

The frequency bands mentioned above are well suited for area coverage, including outdoor-to-indoor and indoor-to-indoor links. Similar frequencies may be used in fixed local access systems (point-to-point and point-to-multipoint) where identical effects can be expected, the main difference being that the channel variability and time spreading will be much smaller.

The wireless channel, in the same way as the various wired channels (optical fiber, coaxial, waveguides, twisted pair, power line, etc.), should provide a distortion-free link between the transmitter and the receiver. This is achieved if the magnitude of its frequency response is flat and the phase is linear, i.e.,

$$
|H(f)|=\text { constant and } \arg (H(f)) \alpha f
$$


where $\alpha$ means proportional to. Equivalently, in the time domain, the associated impulse response should be of the form

$$
h(t)=a \exp (\mathrm{j} \xi) \delta(t-\tau)
$$

where $\xi$ is the phase. The following Fourier transform pair holds,

$$
h(t)=a \exp (\mathrm{j} \xi) \delta(t-\tau) \stackrel{F}{\longleftrightarrow} H(f)=a \exp (\mathrm{j} \xi) \exp (-\mathrm{j} \omega \tau)
$$

where the specifications for a distortion-free channel are fulfilled.

Signals transmitted through the radio channel use a limited portion of the spectrum, small in comparison with the central frequency or the carrier, $f_{\mathrm{c}}$. Such signals are called pass-band signals and follow the expression

$$
y(t)=a(t) \cos \left[2 \pi f_{\mathrm{c}} t+\theta(t)\right]
$$

where $a(t)$ is the envelope of $y(t)$ and $\theta(t)$ is the phase. As all the transmitted information (modulation) is contained in the envelope and the phase, the signal can be analyzed by just using the so-called complex envelope or low-pass equivalent of $y(t)$, given by

$$
r(t)=a(t) \exp [\mathrm{j} \theta(t)]
$$

It will always be possible to recover $y(t)$ from $r(t)$ by simple multiplication by the carrier phasor and taking the real part, i.e.,

$$
y(t)=\operatorname{Re}\left[r(t) \exp \left(\mathrm{j} 2 \pi f_{\mathrm{c}} t\right)\right]
$$

Figure 1.1 shows how the complex envelope can be regarded as a version of the pass-band (RF) signal shifted to a central frequency equal to $0 \mathrm{~Hz}$. The spectrum of the passband signal, $Y(f)$, can be put in terms of the complex envelope spectra, $R(f)$, using the expression [1]

$$
Y(f)=\frac{1}{2}\left[R\left(f-f_{\mathrm{c}}\right)+R^{*}\left(-f-f_{\mathrm{c}}\right)\right]
$$

where $R(f)$ is the Fourier transform of $r(t)$, and * indicates complex conjugate.

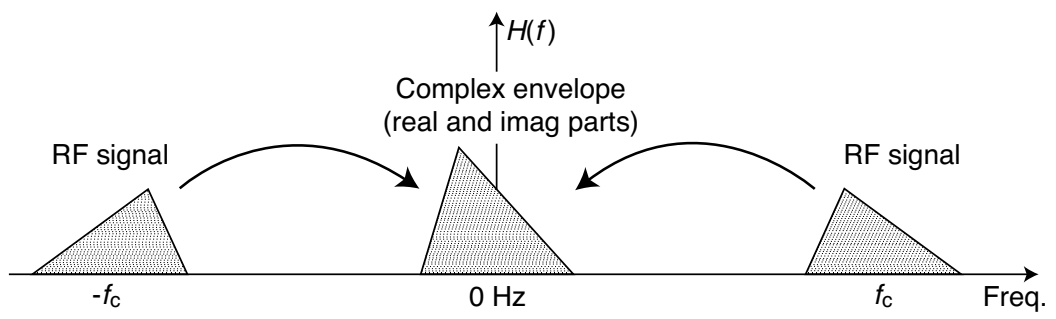

Figure 1.1 Signal spectrum in the band-pass and low-pass domains 
The complex envelope, $r(t)$, instead of being represented in terms of its magnitude and phase, can also be put in terms of its in-phase and quadrature components, i.e.,

$$
r(t)=a(t) \exp [\mathrm{j} \theta(t)]=I(t)+\mathrm{j} Q(t)
$$

In our simulations we will always work in the low-pass equivalent domain.

The complex envelope is in voltage units. To compute the instantaneous power, its magnitude has to be squared, i.e.,

$$
p(t)=\frac{1}{2} \frac{|r(t)|^{2}}{R}
$$

where $R$ is the resistance of the load.

We can try and find the average received power when an unmodulated, continuous wave (CW) is transmitted. The squared rms value of the RF signal voltage can be linked to the rms squared value of the magnitude of complex envelope, thus [1]

$$
y_{\mathrm{rms}}^{2}=\overline{y^{2}(t)}=\frac{1}{2} \overline{|r(t)|^{2}}=\frac{1}{2} r_{\mathrm{rms}}^{2}
$$

where $\bar{x}$ (overbar) means time average of $x$. The average power is given by

$$
\bar{p}=\frac{y_{\mathrm{rms}}^{2}}{R}=\frac{\overline{y^{2}(t)}}{R}=\frac{\overline{|r(t)|^{2}}}{2 R}=\frac{r_{\mathrm{rms}}^{2}}{2 R}
$$

where $\bar{p}$ is the average power. We will be making reference later in this chapter to the rms value of the complex envelope for the Rayleigh case (Project 1.1).

Unfortunately, the channel response does not remain constant in time as the terminal moves. Even when the terminal is stationary, time variations may arise. The channel can also introduce distortion, frequency shifts and other effects as we will be discussing in this and other chapters.

Furthermore, the frequency spectrum is not used exclusively by the wanted link: adjacent frequencies, or even the same frequency, are used in an ever increasingly crowded spectrum. Thus, it is important to perform a joint analysis of the wanted link and interfering links. This point will be discussed in some detail in Chapter 3.

This book will be reproducing the characteristics of the wanted and interfering signals, especially their dynamics. Thus, time series will be generated in most of the projects proposed throughout. Sometimes, the term 'time series' will be used indistinctly for 'series' with the abscissas in time units or in length units (traveled distance), thus explicitly showing the time and/or location variability in the received signal or in the propagation channel parameters of interest: power, voltage, phase, attenuation, frequency response, etc.

The procedure to be followed in all chapters will be to first define a propagation scenario, either statistically or geometrically, then generate one or several time series and, finally, perform a statistical analysis of the produced time series for extracting relevant parameters from which conclusions may be drawn. The only exception is in this chapter where we will be using series already produced using simulators from other chapters. We will be analyzing these series in order to get acquainted with some of the basic techniques that will be used throughout the book. 


\subsection{Wireless Propagation Basics}

Now, we would like to briefly discuss some of the characteristics of the mobile/wireless propagation channel [2]. The modeling techniques involved in land mobile systems have also many similarities with those used in other area coverage systems such as sound and TV broadcasting. The convergence of two-way and broadcast, as well as of mobile and fixed systems is making such systems almost indistinguishable, as they try to aim at the same users with offerings of similar services. The similarities between fixed and mobile wireless channels over the frequency bands of interest not only include the mechanisms giving rise to path loss, but also they are subjected to shadowing and multipath effects even though these are normally much milder.

Depending on the location and the BS or access point height, cells of larger or smaller size can be created. The classical cellular environment of tall masts above rooftops gives rise to so-called macrocells. Propagation in these conditions will take up most of the discussion in this chapter and most of this book. As the BS antenna height becomes smaller and is below the surrounding rooftops, so-called microcells are generated. BSs within buildings give rise to picocells (Chapter 8). However, when satellites are used, which means much higher 'BS antenna heights', megacells are originated (Chapter 9).

Man-made structures [3] such as buildings or small houses in suburban areas, with sizes ranging from a few meters to tens of meters, dramatically influence the wireless propagation channel. In urban areas, the size of structures can be even larger. Likewise, in rural and suburban environments, features such as isolated trees or groups of trees, etc. may reach similar dimensions. These features are similar or greater in size than the transmitted wavelength (metric, decimetric, centimetric waves) and may both block and scatter the radio signal causing specular and/or diffuse reflections. These contributions may reach MS by way of multiple paths, in addition to that of the direct signal. In many cases, these echoes make it possible that a sufficient amount of energy reaches the receiver, so that the communication link is feasible. This is especially so when the direct signal is blocked. Hence, in addition to the expected distance power decay, two main effects are characteristic in mobile propagation: shadowing and multipath.

We can identify three levels in the rate of change of the received signal as a function of the distance between BS and MS, namely, very slow variations due to range, slow or long-term variations due to shadowing and fast or short-term variations due to multipath.

While in conventional macrocells, BS heights are in the order of $30 \mathrm{~m}$ and are normally set on elevated sites with no or few blocking/scattering elements in their surroundings, MS antenna heights are usually smaller than those of local, natural and man-made features. Typical values range from 1.5 or so for handheld terminals to $3 \mathrm{~m}$ for vehicular terminals. For other radiocommunication systems for TV broadcasting or fixed wireless access operating in the same frequency bands, the propagation channel will present a milder behavior given that, in these cases, the receive antennas are usually directive and are normally sited well above the ground. Both the shadowing effect on the direct signal and the amount of multipath is considerably reduced.

Other operating scenarios where both ends of the link are surrounded by obstacles are in indoor communications where walls, the ceiling or the various pieces of furniture will clearly determine the propagation conditions. 
The frequencies used in mobile communications are normally above $30 \mathrm{MHz}$ and the maximum link lengths do not exceed 25 to $30 \mathrm{~km}$. Macrocells in current $2 \mathrm{G}$ (second generation, e.g., GSM) or 3G (third generation, e.g., UMTS) systems are much smaller. It must be taken into account that mobile communications are two-way and that the uplink (MS to BS) is power limited. This is especially so in the case of regular portable, handheld terminals. Furthermore, mobile system coverage ranges are short due to the screening effects of the terrain and buildings in urban areas. This makes frequency reuse possible at relatively short distances. This is also an important feature in mobile networks which require a great spectral efficiency for accommodating larger and larger numbers of users.

Currently $3 \mathrm{G}$ wireless systems are being deployed in the $2 \mathrm{GHz}$ band while wireless LANs are beginning to be deployed in the $5 \mathrm{GHz}$ band while, still, the $2.4 \mathrm{GHz}$ band is the most popular for this application. Fixed access systems in licensed bands with ranges of several $\mathrm{km}$ to a few tens of $\mathrm{km}$ are being deployed in the $3.5 \mathrm{GHz}$ band in Europe while in the Americas their assigned band is closer to $2 \mathrm{GHz}$. The $5 \mathrm{GHz}$ band will also be used in unlicensed fixed access network applications. Very promising, short-range systems are being proposed at higher frequencies such as in the neighborhood of $60 \mathrm{GHz}$ where gaseous absorption mechanisms dominate. Such phenomena are not dealt with in this book.

Two representative and extreme scenarios may be considered:

(a) the case where a strong direct signal is available together with a number of weaker multipath echoes, i.e., line-of-sight (LOS) conditions; and

(b) the case where a number of weak multipath echoes is received and no direct signal is available, non line-of-sight (NLOS) conditions.

Case (a) occurs in open areas or in very specific spots in city centers, in places such as crossroads or large squares with a good visibility of BS. Sometimes, there might not be a direct LOS signal but a strong specular reflection off a smooth surface such as that of a large building will give rise to similar conditions. This situation may be modeled by a Rice distribution for the variations of the received RF signal envelope: the Rice case. Under these conditions, the received signal will be strong and with moderate fluctuations (Figure 1.2). The Rice distribution is studied in Chapters 5 and 6.
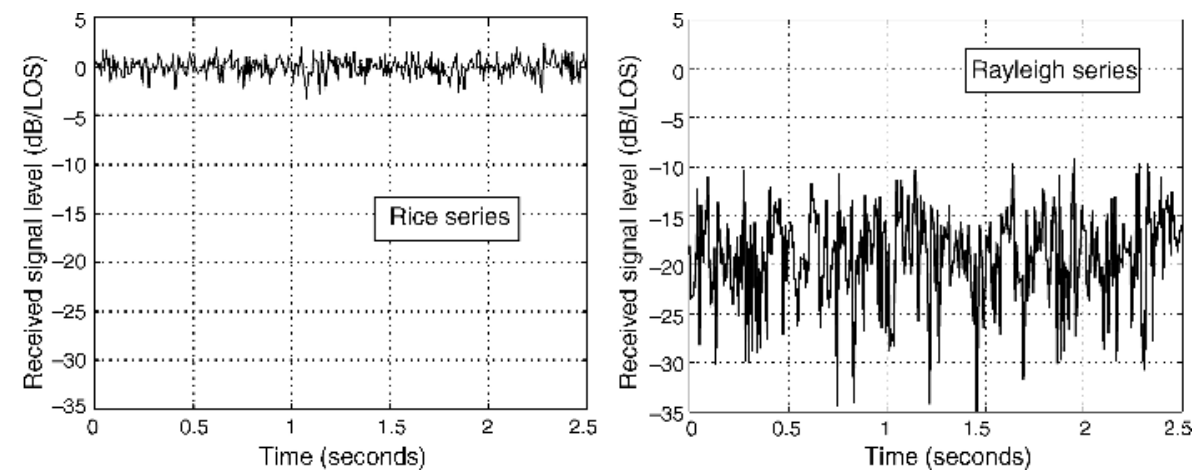

Figure 1.2 Rice and Rayleigh distributed time series. Frequency $900 \mathrm{MHz}$, mobile speed $10 \mathrm{~m} / \mathrm{s}$ 


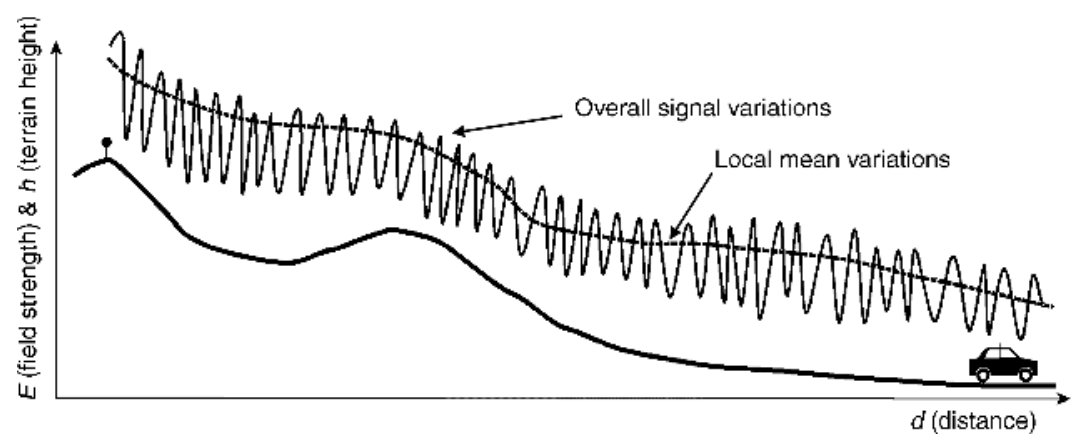

Figure 1.3 Variations in the received signal with the movement of the mobile [2]

Case (b) will typically be found in highly built-up urban environments. This is a worstcase scenario since the direct signal is completely blocked out and the overall received signal is only due to multipath, thus being weaker and subjected to marked variations (Figure 1.2). This kind of situation may also occur in rural environments where the signal is obstructed by dense masses of trees: wooded areas or tree alleys. The received signal amplitude variations in this situation are normally modeled with a Rayleigh distribution: the Rayleigh case.

The received field strength or the received voltage may be represented in the time domain, $r(t)$, or in the traveled distance domain, $r(x)$. Figure 1.3 shows a typical mobile communications scenario with MS driving away from BS along a radial route so that the link profile is the same as that of the route profile. The figure also shows a sketch of the received signal as a function of the distance from BS. The first thing to be noted is that the signal is subjected to strong oscillations as MS travels away from BS. The three rates of signal variation are also schematically represented.

For carrying out propagation channel measurements, the mobile speed, $V$, should remain constant. Of course, there are ways around this. In such cases, the traversed distance needs to be recorded too. In our simulations in later chapters and in the series analyzed in this chapter, we will assume a constant MS speed. For a constant $V$, it is straightforward to make the conversion between the representation in the time, $r(t)$, and traveled distance domains, $r(x)(t=x / V)$.

Variable $x$ may either be expressed in meters or in wavelengths. Based on such signal recordings plotted in the distance domain, it is possible to separate and study individually the fast and slow variations, due respectively to multipath and shadowing, as illustrated in Figure 1.4.

Generally, the received signal variations, $r(t)$ or $r(x)$, may be broken down, in a more or less artificial way, into two components [3]

- the slow or long-term variations: $m(t)$ or $m(x)$; and

- the fast or short-term variations: $r_{0}(t)$ or $r_{0}(x)$.

The received signal may, therefore, be described as the product of these two terms,

$$
r(t)=m(t) \cdot r_{0}(t) \text { or, alternatively, } r(x)=m(x) \cdot r_{0}(x)
$$



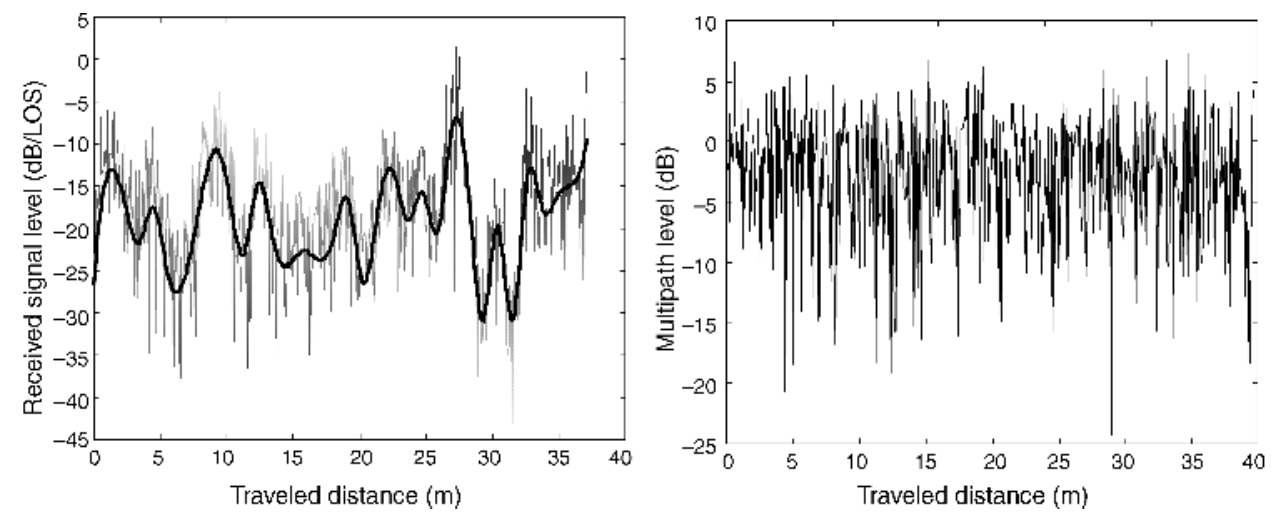

Figure 1.4 Overall and slow variations, and fast variations after removing the slow variations

when expressed in linear units. In $\mathrm{dB}$, the products become additions, i.e.,

$$
R(t)=M(t)+R_{0}(t) \quad \text { or, alternatively, } \quad R(x)=M(x)+R_{0}(x)
$$

With this approach we are assuming that the fast variations are superposed on the slow variations. Figure 1.4 illustrates an overall time series where the slow variations are also plotted. The figure also shows the fast variations after removing (filtering out) the slow variations. The slow variations can be extracted from the overall variations through low-pass filtering by computing a running mean. This is equivalent to calculating the signal average for the samples within a route section of length $2 L$ equal to some tens of wavelengths,

$$
m\left(x_{i}\right)=\frac{\sum_{k=-N}^{N} r_{i+k}}{2 N+1} \text { for } \quad r_{i-N} \ldots r_{i} \ldots r_{i+N} \in x_{i}-L<x<x_{i}+L
$$

Typically, lengths of $10 \lambda$ to $40 \lambda$ are used [3]. For example, for the $2 \mathrm{GHz}(\lambda=0.15 \mathrm{~m})$ band used in $3 \mathrm{G}$ mobile communications, the averaging length would be $2 L \approx 3-6 \mathrm{~m}$. The average value, $m\left(x_{i}\right)$, computed for a given route position $x_{i}$ is usually called the local mean at $x_{i}$.

It has been observed experimentally [3] that the slow variations of the received signal, that is, the variations of the local mean, $m(x)$, follow a lognormal distribution (Chapter 6) when expressed in linear units $(\mathrm{V}, \mathrm{V} / \mathrm{m}, \ldots)$ or, alternatively, a normal distribution when expressed in logarithmic units, $M\left(x_{i}\right)=20 \log m\left(x_{i}\right)$.

The length, $2 L$, of route considered for the computation of the local mean, i.e., used to separate out the fast from the slow variations, is usually called a small area or local area. It is within a small area where the fast variations of the received signal are studied since they can be described there with well-known distributions (Rayleigh).

Over longer route sections ranging from $50 \mathrm{~m}$ or $100 \mathrm{~m}$ to even 1 or $2 \mathrm{~km}$, the variations of the local mean are generally studied. This extended surface is usually called a larger area. Typically, standard propagation models do not attempt to predict the fast signal variations. 
Instead they predict the mean, $\bar{M}(x)=\bar{E}(x)$, or, $\bar{M}(x)=\bar{V}(x)$, and the standard deviation (or location variability) $\sigma_{\mathrm{L}}$ of the local mean variations (normal distribution in $\mathrm{dB}$ ) within the larger area.

Before low-pass filtering, the very slow variations due to the distance from BS must be removed. Free-space loss, $L_{\mathrm{fs}}(\mathrm{dB})$, is a very common model for the range-dependent loss. It is given by

$$
l_{\mathrm{fs}}=\frac{p_{\mathrm{t}}}{p_{\mathrm{r}}}=\left(\frac{4 \pi d}{\lambda}\right)^{2}
$$

or, in practical units,

$$
L_{\mathrm{fs}}(\mathrm{dB})=32.4+20 \log f(\mathrm{MHz})+20 \log d(\mathrm{~km})
$$

where we have assumed isotropic antennas, i.e., with $0 \mathrm{~dB}$ gain or unit gain in linear units, with $p_{\mathrm{t}}$ and $p_{\mathrm{r}}$ the transmitted and received powers in $\mathrm{W}$, and $L_{\mathrm{fs}}$ and $l_{\mathrm{fs}}$ the free-space loss in $\mathrm{dB}$ and in linear units (power ratio), respectively. Throughout this book, variables in capital letters will normally denote magnitudes expressed in logarithmic units $(\mathrm{dB})$ and lower case letters will denote magnitudes expressed in linear units.

The free-space loss gives rise to a distance decay in the received power following an inverse power law of exponent $n=2$ (Figure 1.5), i.e.,

$$
p_{1} \alpha \frac{1}{d_{1}^{2}} \quad \text { and } \quad p_{2} \alpha \frac{1}{d_{2}^{2}} \quad \text { then, in } \mathrm{dB}, \quad \Delta_{\mathrm{p}}=10 \log \frac{p_{2}}{p_{1}}=20 \log \frac{d_{1}}{d_{2}}
$$

where $\Delta_{\mathrm{p}}$ is the received power difference in $\mathrm{dB}$. The above expressions show a $20 \mathrm{~dB} /$ decade $(20 \mathrm{~dB}$ decrease when the distance is multiplied by 10$)$ or $6 \mathrm{~dB} /$ octave $(6 \mathrm{~dB}$ decrease when the distance is doubled) distance decay rate.

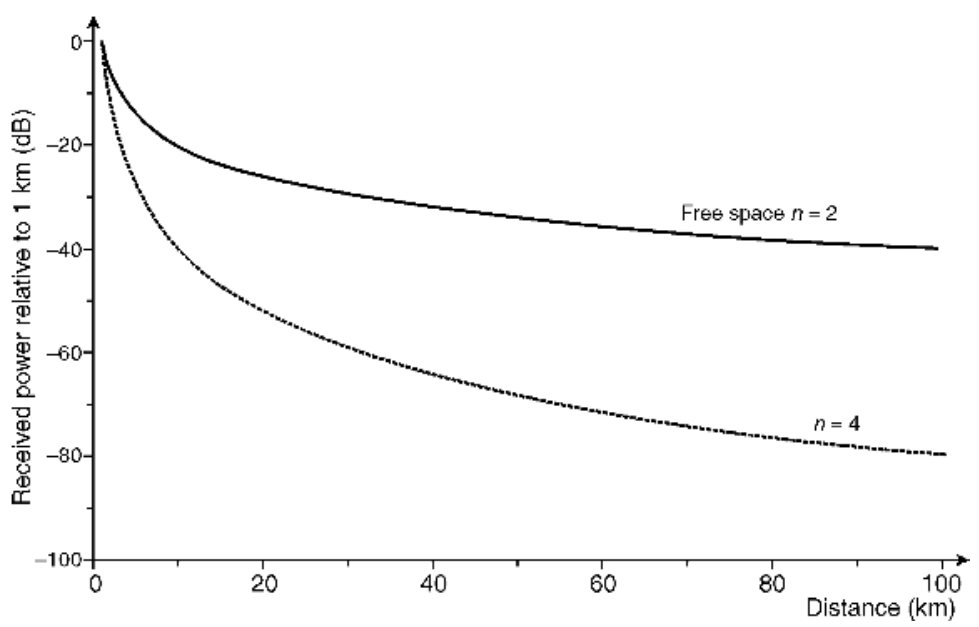

Figure 1.5 Received signal decay with distance: $n=2$ and $n=4$ laws 
These variations are steeper in the first few kilometers of the radio path and become gentler for the longer distances. For example, using Equation 1.16, from $\mathrm{km} 1$ to $\mathrm{km} \mathrm{2}$, a $6 \mathrm{~dB}$ decrease takes place. However, the same $6 \mathrm{~dB}$ reduction is observed from $\mathrm{km} 10$ to $\mathrm{km} 20$.

It has been experimentally observed that, in typical mobile propagation paths, the signal's distance decay does not follow an $n=2$ power law (as in free space) but, rather, it presents a larger exponent. Signal decay is usually modeled by an $l \alpha d^{n}$ law, i.e., $l$ being proportional to the distance risen to the power $n$. The values of $n$ typically are somewhere near 4, i.e., $40 \mathrm{~dB} /$ decade (Figure 1.5). Widespread propagation models, briefly discussed in Chapter 3 such as those of Hata [4] and COST 231 [5], also predict exponents close to 4.

The path-loss expressions normally provided by propagation models (Chapter 3 ) are of the form

$$
L(\mathrm{~dB})=A+B \log d(\mathrm{~km})=A+10 n \log d(\mathrm{~km})
$$

where $A$ and $n$ are dependent on the frequency and a number of other factors as listed below. Parameter $A$ is the loss at a reference distance, in this case, $1 \mathrm{~km}$, and $n$ is the propagation decay law. Several factors, apart from the frequency and the distance that influence path loss, are taken into consideration by existing propagation models affecting the expressions for $A$ and $n$. These factors are:

- the height of the MS antenna;

- the height of the BS relative to the surrounding terrain (effective height);

- the terrain irregularity (sometimes called undulation, $\Delta h$, or roughness, $\sigma_{\mathrm{t}}$ );

- the land usage in the surroundings of MS: urban, suburban, rural, open, etc.

When calculating the path loss, all such factors must be taken into account, i.e.,

$$
L=L_{\text {Reference }}+L_{\text {Terrain Irregularity }}+L_{\text {Environment }}
$$

The path loss is defined as that existing between isotropic antennas ( $0 \mathrm{~dB}$ gain $)$. Isotropic antennas do not exist in practice but are commonly used in link budget calculations since they allow the definition of the loss independently of the antenna type. Then, when computing an actual link budget, the gains of the antennas to be used must be introduced in the calculations.

The path loss is made up of three main components: a reference loss, typically the freespace loss, although some models like Hata's [4] take the urban area loss as reference. Other models [6] use the so-called plane-earth loss $(n=4)$ as their reference (Chapter 8).

The second component is the loss due to terrain irregularity (we will be discussing this in some detail in Chapter 2) and, finally, the third component is the loss due to the local clutter or local environment where the additional loss will very much depend on the land usage in the vicinity of MS: urban, suburban, rural, open, woodland, etc.

\subsection{Link Budgets}

Here we briefly remind the reader of some of the basic elements in link budget calculations. In fact, link budgets are not only used for the wanted signal but also for noise and 
the interference. The equations below are valid for the wanted and the interference signals.

The path loss relates the transmit and received powers assuming no transmitter/receiver loss or gain (between isotropic antennas), i.e.,

$$
L(\mathrm{~dB})=10 \log \left(p_{\mathrm{t}} / p_{\mathrm{r}}\right)
$$

The path loss can be split into the sum of the free-space loss and the so-called excess loss,

$$
L(\mathrm{~dB})=L_{\mathrm{fs}}+L_{\text {excess }}
$$

where the excess loss is given by,

$$
L_{\text {excess }}(\mathrm{dB})=20 \log \left(e_{0} / e\right)
$$

where $e_{0}$ is the field strength at the received antenna under free-space conditions and $e$ is the actual field strength both in linear units $(\mathrm{V} / \mathrm{m})$.

So far we have assumed that the link does not contain gains or losses other than the path loss. If the gains and losses at both ends of the link are taken into account, the received power is given by

$$
p_{\mathrm{r}}=\frac{p_{\mathrm{t}} g_{\mathrm{t}} g_{\mathrm{r}}}{l_{\mathrm{t}} l_{\mathrm{r}}}
$$

given in linear units or, in logarithmic units,

$$
P_{\mathrm{r}}(\mathrm{dBW} \text { or } \mathrm{dBm})=P_{\mathrm{t}}+G_{\mathrm{t}}+G_{\mathrm{r}}-L_{\mathrm{t}}-L-L_{\mathrm{r}}
$$

where $l_{\mathrm{t}}$, or alternatively, $L_{\mathrm{t}}$ when using $\mathrm{dB}$, is the loss at the transmit side, e.g., cables, etc., and $g_{\mathrm{t}}\left(G_{\mathrm{t}}\right)$ is the transmit antenna gain.

A frequently used parameter for describing the radiated power is the EIRP (equivalent isotropic radiated power) defined as

$$
\operatorname{eirp}=p_{\mathrm{t}} g_{\mathrm{t}} / l_{\mathrm{t}}
$$

in linear units or, in $\mathrm{dB}$,

$$
\operatorname{EIRP}(\mathrm{dBW} \text { or } \mathrm{dBm})=P_{r}+G_{t}-L_{T}
$$

Link budgets are usually computed in terms of the received power. However, in some cases, the field strength $(\mathrm{V} / \mathrm{m})$ or the power flux density $\left(\mathrm{W} / \mathrm{m}^{2}\right)$ are of interest, especially in interference studies. The received power can also be put in terms of the power flux density, $\phi$, and the effective antenna aperture, $A_{\mathrm{e}}\left(\mathrm{m}^{2}\right)$, i.e.,

$$
p_{\mathrm{r}}=\phi \cdot A_{\mathrm{e}}
$$

where the effective antenna aperture is related to the gain through

$$
g_{\mathrm{r}}=\frac{4 \pi}{\lambda^{2}} A_{\mathrm{e}}
$$


where $g_{\mathrm{r}}$ is in linear units (power ratio). In $\mathrm{dB}, G_{\mathrm{r}}(\mathrm{dB})=10 \log \left(g_{\mathrm{r}}\right)$. The gain is frequently given with reference to an isotropic antenna and instead of $\mathrm{dB}, \mathrm{dBi}$ units are often used. We will use both denominations indistinctly.

Coming back to the power flux density, it can be given in terms of the field strength at the receive antenna,

$$
\phi=\frac{1}{2} \frac{|e|^{2}}{\eta}=\frac{1}{2} \frac{|e|^{2}}{120 \pi} \mathrm{W} / \mathrm{m}^{2}
$$

where peak field strength values are used. If rms values were used the $1 / 2$ in the right-hand side would be dropped. The term $\eta$ is the impedance of free space which is equal to $120 \pi$. The power density at the receive antenna can also be put in terms of the transmitted power or the EIRP,

$$
\phi=\frac{p_{\mathrm{t}} g_{\mathrm{t}} / l_{\mathrm{t}}}{4 \pi d^{2}}=\frac{\text { eirp }}{4 \pi d^{2}} \mathrm{~W} / \mathrm{m}^{2}
$$

By equating the two expressions for $\phi$, we can find the expression for the field strength at the receive antenna under free-space conditions,

$$
\frac{\text { eirp }}{4 \pi d^{2}}=\frac{1}{2} \frac{|e|^{2}}{120 \pi} \rightarrow|e|=\frac{\sqrt{60 \text { eirp }}}{d}(\mathrm{~V} / \mathrm{m})
$$

If the rms field strength is wanted, we should replace $\sqrt{60}$ by $\sqrt{30}$.

Finally, the well-known Friis equation for free-space conditions linking the transmit and receive powers can be reached by developing further Equation 1.27, thus,

$$
p_{\mathrm{r}}=\phi A_{\mathrm{e}}=\frac{p_{\mathrm{t}} g_{\mathrm{t}} / l_{\mathrm{t}}}{4 \pi d^{2}} \frac{\lambda^{2}}{4 \pi} g_{\mathrm{r}} / l_{\mathrm{r}}
$$

then

$$
\frac{p_{\mathrm{r}}}{p_{\mathrm{t}}}=\frac{g_{\mathrm{t}} g_{\mathrm{r}}}{l_{\mathrm{t}} l_{\mathrm{r}}}\left(\frac{\lambda}{4 \pi d}\right)^{2}=\frac{g_{\mathrm{t}} g_{\mathrm{r}}}{l_{\mathrm{t}} l_{\mathrm{r}} l_{\mathrm{fs}}}
$$

where $l_{\mathrm{fs}}$ is the free-space loss given in Equation 1.15. In the above expressions we should include other terms such as those due to impedance or polarization mismatch when necessary.

Two other link budgets associated with two basic propagation mechanisms are also presented next: one corresponds to specular reflections and the other to diffuse scattering on small objects, or on non-specular objects or rough surfaces such as the terrain. Other propagation mechanisms such as diffraction are dealt with in some detail in Chapter 2.

When a surface such as the ground or a building face is large and smooth, specular reflections can take place and reach the receiver provided that Snell's law is fulfilled, i.e., 
the angle of incidence and the angle of reflection are equal. In this case, the received power is ruled by the formula

$$
\frac{p_{\mathrm{r}}}{p_{\mathrm{t}}}=\left[\frac{\lambda|R|}{4 \pi\left(d_{1}+d_{2}\right)}\right]^{2}
$$

where the magnitude of Fresnel's reflection coefficient for the relevant polarization is included. Later in Chapter 8, the expressions of the complex reflection coefficients for the vertical and horizontal polarizations are given. For a power budget, the phase of the reflection coefficient is not needed, only its absolute value squared. However, if several rays combine coherently at the receiver their phases must be accounted for.

When the scattering object is not flat and smooth, or it is small, it will not show the same properties of specular reflectors. In this case, the scattered energy is shed in all directions or, possibly, within a given angular sector. Here, the link budget for diffuse reflections on this type of obstacles is ruled by the bistatic radar equation,

$$
\frac{p_{\mathrm{r}}}{p_{\mathrm{t}}}=\frac{g_{\mathrm{t}}}{l_{\mathrm{t}}} \frac{1}{4 \pi d_{1}^{2}} \sigma \frac{1}{4 \pi d_{2}^{2}} \frac{\lambda^{2}}{4 \pi} \frac{g_{\mathrm{r}}}{l_{\mathrm{r}}}
$$

where the obstacle parameter is its radar cross-section, $\sigma\left(\mathrm{m}^{2}\right)$.

This propagation mechanism produces much smaller contributions than those of specular reflections even for very large values of $\sigma$. This is due to the fact that two squared distances appear in its link budget in lieu of one distance (more specifically, the sum of two distances) as in the specular reflection case. Throughout this book, when modeling multipath, we will be using a multiple point-scatterer model. The powers for their corresponding contributions should fulfill the bistatic radar equation.

So far we have discussed static power budgets. In fact, the received signals are time varying. To account for such variations, when computing the system outage probability or setting up fade margins, the distribution of the variability, fast and slow, of the received signal must be well known. A good statistical knowledge of the signal variability behavior is of paramount importance for an optimal system planning. In addition, there is also the variability in the various interference sources (Chapter 3) and the noise.

\subsection{Projects}

We have reviewed in a concise way some relevant issues in wireless propagation modeling, especially those related to path loss and signal variability. Other concepts will be presented later in the book but, for the time being, this should be enough to get started. Now, we want to address some basic analysis techniques for time series, either measured or synthesized. We will do this on a step-by-step basis by introducing new concepts with each new project.

\section{Project 1.1: Fast Fading Series}

In this project, series11.mat (Figure 1.6) is supplied for analysis. It corresponds to a short section of simulated signal (in $\mathrm{dBm}$ ) assumed to be received under homogeneous multipath conditions. This series, even though simulated, could just as well be a measured one. 


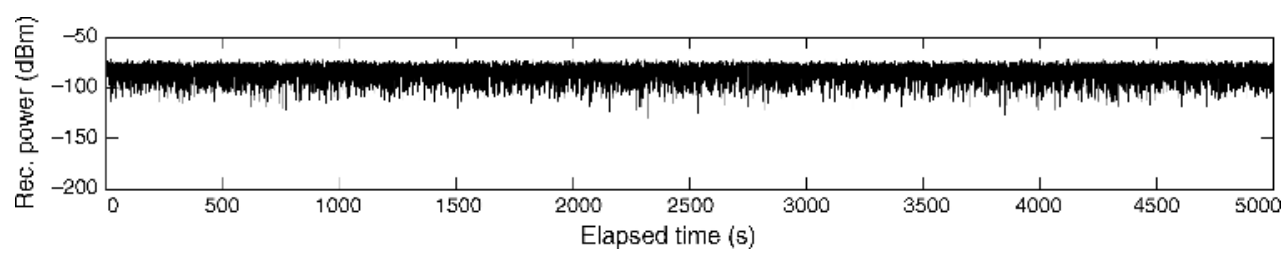

Figure 1.6 series11 processed in Project 1.1

One possible way of recording a measured series could be in $\mathrm{dB}$ units, e.g., $\mathrm{dBm}$ ( $\mathrm{dB}$ relative to $1 \mathrm{~mW}$ ) as is the case here. This could correspond to a recording with a spectrum analyzer or field strength meter. In other cases, the measured series could be given in terms of analog to digital converter (ADC) units which must be translated into voltage or power units. In other cases, as in Project 1.3, the series can be given in terms of its in-phase and quadrature components. File series11. mat contains a two-column matrix which includes, in the first column, the time axis in seconds and, in the second column, the power in $\mathrm{dBm}$.

Signal variations caused by multipath, in the case where the direct signal is assumed to be totally blocked, are usually represented by a Rayleigh distribution when expressed in units of voltage. We will see later in Chapter 5 how, if the magnitude were the power, we would have an exponential distribution. The probability density function, pdf, of the Rayleigh distribution is given by

$$
f(r)=\frac{r}{\sigma^{2}} \exp \left(-\frac{r^{2}}{2 \sigma^{2}}\right) \quad \text { for } r \geq 0
$$

where $r$ is a voltage which actually represents, $|r|$, the magnitude of the complex envelope. We have dropped the magnitude operator for simplicity. This distribution has a single parameter, its mode or modal value, $\sigma$. Other related parameters are given in Table 1.1 as a function of $\sigma$. Script intro11 is provided, which was used for plotting the Rayleigh pdf in Figure 1.7.

By integrating the pdf, the cumulative distribution function, $\mathrm{CDF}$, can be obtained, i.e.,

$$
\operatorname{CDF}(R)=\operatorname{Prob}(r \leq R)=\int_{0}^{R} f(r) d r=1-\exp \left(-\frac{R^{2}}{2 \sigma^{2}}\right)
$$

Table 1.1 Rayleigh distribution parameters as a function of its mode

\begin{tabular}{ll}
\hline Mode & $\sigma$ \\
Median & $\sigma \sqrt{2 \ln 2}=1.18 \sigma$ \\
Mean & $\sigma \sqrt{\pi / 2}=1.25 \sigma$ \\
RMS value & $\sigma \sqrt{2}=1.41 \sigma$ \\
Standard deviation & $\sigma \sqrt{2-\pi / 2}=0.655 \sigma$ \\
\hline
\end{tabular}




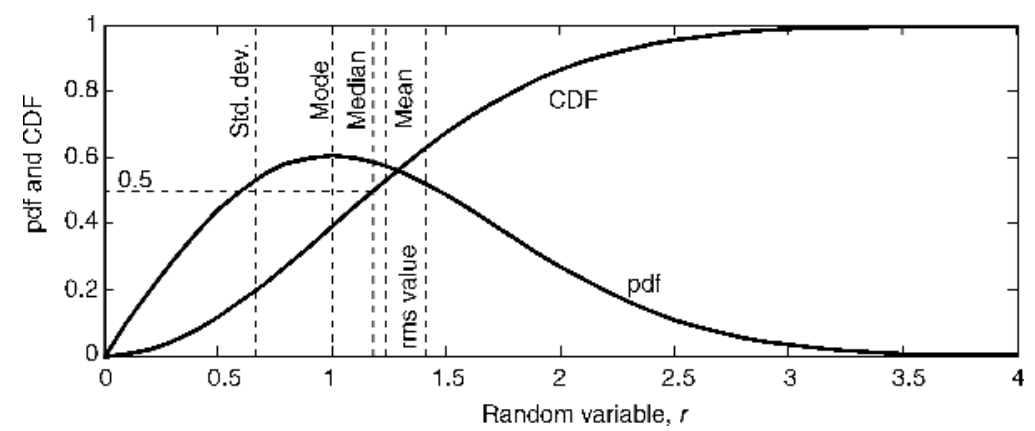

Figure 1.7 Probability density function and cumulative distribution function for a Rayleigh distribution with $\sigma=1$. Generated with intro11

Rayleigh pdf and CDF functions are plotted in Figure 1.7 for $\sigma=1$. The CDF is very useful when computing outage probabilities in link budgets. The CDF gives the probability that a given signal level is not exceeded. If this level is the system's operation threshold, this provides us with the probability that the signal level is equal or below such threshold, i.e., the outage probability. Knowing the CDF adequate fade margins can also be set up.

The parameters in Table 1.1 are defined as follows:

$$
\begin{gathered}
\operatorname{mean}(r)=E[r]=\int_{-\infty}^{\infty} r f(r) d r=\sigma \sqrt{\frac{\pi}{2}}=1.2533 \sigma \\
\operatorname{rms}^{2}(r)=E\left[r^{2}\right]=\int_{-\infty}^{\infty} r^{2} f(r) d r=2 \sigma^{2} \\
\operatorname{variance}(r)=E\left[r^{2}\right]-E[r]^{2}=2 \sigma^{2}-\frac{\sigma^{2} \pi}{2}=\sigma^{2}\left(\frac{4-\pi}{2}\right)=0.4292 \sigma^{2} \\
1-\exp \left(-\frac{\tilde{r}^{2}}{2 \sigma^{2}}\right)=0.5, \text { thus, median }(r)=\tilde{r}=\sqrt{2 \sigma^{2} \ln (2)}=1.1774 \sigma
\end{gathered}
$$

where $E[]$ is the expectation operator.

What we will do in this project (script project11) is analyze series11 by computing its histogram (approximation of its pdf) and its sample CDF, and we will verify whether the series provided fits a Rayleigh distribution. The series is plotted in $\mathrm{dBm}$ in Figure 1.6. What we want is to model the voltage. A load resistance, $R$, of $50 \Omega$ is assumed, thus, $p(\mathrm{~W})=1000 \times 10^{P(\mathrm{dBm}) / 10}$. The power and the voltage are linked through $v=\sqrt{2 R p}$.

The resulting values of $v$ are very small and awkward to handle. Hence, for working with voltages, we will normalize the series with respect to one of its parameters. The preferred option here is to keep using the expression for the pdf given above, i.e., as a function of the mode. However, the estimation of the mode from the time series is complicated. We can, however, estimate the mode from the mean in the case of a Rayleigh distribution by using the 


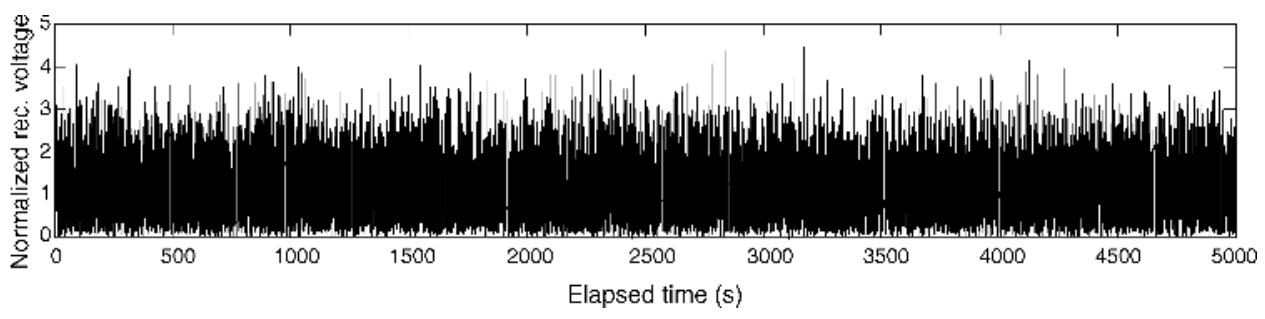

Figure 1.8 Voltage time series normalized with respect to its estimated modal value

equality $\sigma \sqrt{\pi / 2}=1.25 \sigma$ in Table 1.1 . Thus the series of $v$ has been normalized with respect to its estimated modal value, $\sigma$, i.e., $v^{\prime}=v / \sigma$ (Figure 1.8). The new series, $v^{\prime}$, should have a modal value equal to one. Another alternative would have been to use a version of the Rayleigh distribution put as a function of the mean. This would involve a very simple change of variable as shown below. The results would have been identical.

For completeness we show how the Rayleigh distribution can be expressed in terms of several of its parameters other than its mode. As a function of the mean the pdf and CDF are as follows,

$$
f(r)=\frac{\pi r}{2 \bar{r}^{2}} \exp \left(-\frac{\pi r^{2}}{4 \bar{r}^{2}}\right) \quad \text { for } \quad r \geq 0 \quad \text { and } \quad P(R)=1-\exp \left(-\frac{\pi R^{2}}{4 \bar{r}^{2}}\right)
$$

where $\bar{r}$ is the mean. Put now as a function of the rms value, the pdf and CDF have the form,

$$
f(r)=\frac{2 r}{\overline{r^{2}}} \exp \left(-\frac{r^{2}}{\overline{r^{2}}}\right) \quad \text { for } \quad r \geq 0 \quad \text { and } \quad P(R)=1-\exp \left(-\frac{R^{2}}{\overline{r^{2}}}\right)
$$

where $\sqrt{\overline{r^{2}}}$ is the rms value. Finally, as a function of the median,

$f(r)=\frac{2 r \ln (2)}{\tilde{r}^{2}} \exp \left(-\frac{r^{2} \ln (2)}{\tilde{r}^{2}}\right) \quad$ for $\quad r \geq 0 \quad$ and $\quad P(R)=1-\exp \left(-\frac{R^{2} \ln (2)}{\tilde{r}^{2}}\right)$

where $\tilde{r}$ is the median of the distribution.

Now we go on to analyze the normalized series in Figure 1.8. Its average value is 1.25 which is equivalent to a modal value equal to one. We want to know whether the normalized series follows a Rayleigh distribution of mode one. In Figure 1.9 we plot the theoretical and the sample CDFs where we can observe that the match is reasonably good. The theoretical CDF has been computed using Equation 1.37 (RayleighCDF) and the sample CDF using function $\mathrm{ECDF}$. Helpful MATLAB ${ }^{\circledR}\left(\mathrm{MATLAB}^{\circledR}\right.$ is a registered trademark of The MathWorks, Inc.) functions are hist and cumsum.

Figure 1.10 shows the theoretical and measured histograms where the theoretical values have been plotted using MATLAB ${ }^{\circledR}$ function bar and the equation

$$
\operatorname{Prob}\left(R_{1}<r<R_{2}\right)=\int_{R_{1}}^{R_{2}} f(r) d r=\exp \left(-\frac{R_{2}^{2}}{2 \sigma^{2}}\right)-\exp \left(-\frac{R_{1}^{2}}{2 \sigma^{2}}\right)
$$




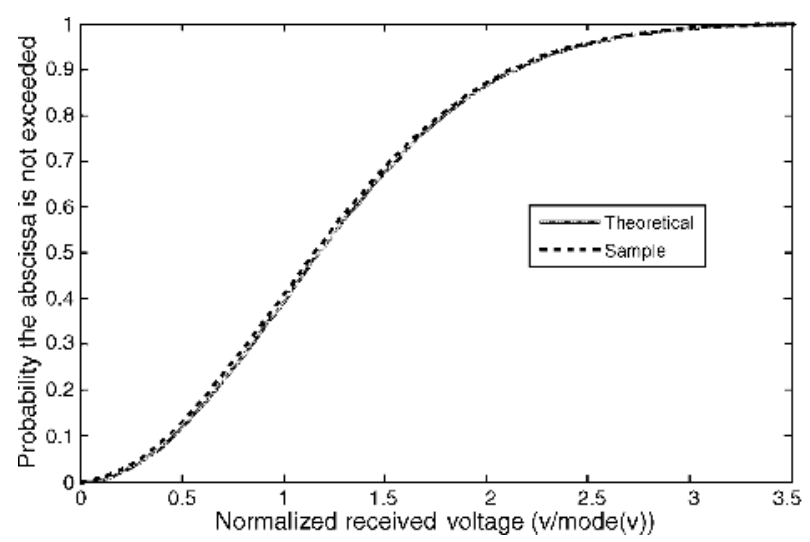

Figure 1.9 Time series and $\sigma=1$ Rayleigh CDFs

where $R_{1}$ and $R_{2}$ are the bin limits (RayleighHIST). Again the match from a visual point of view is quite good. The histogram of the series can be computed using MATLAB ${ }^{\mathbb{R}}$ function hist that provides bin centers and associated frequencies, i.e., the number of occurrences. The frequencies have to be divided by the total number of samples in the series for converting them into probabilities.

Now, we are going to check whether the fit is good enough. As said, from a visual comparison between the measured and the theoretical CDFs and histograms, it is clear that the agreement is quite good. Now we want to quantify how good the fit is. This can be achieved by means of the chi-square goodness-of-fit test [7]. Other tests are also commonly used like the Kolmogorov-Smirnov test [7].

There are two basic elements in the chi-square test [7]. First, we must define a measure of the difference between the values observed experimentally and the values that would be expected if the proposed pdf were correct. Second, this measure has to be compared with $a$

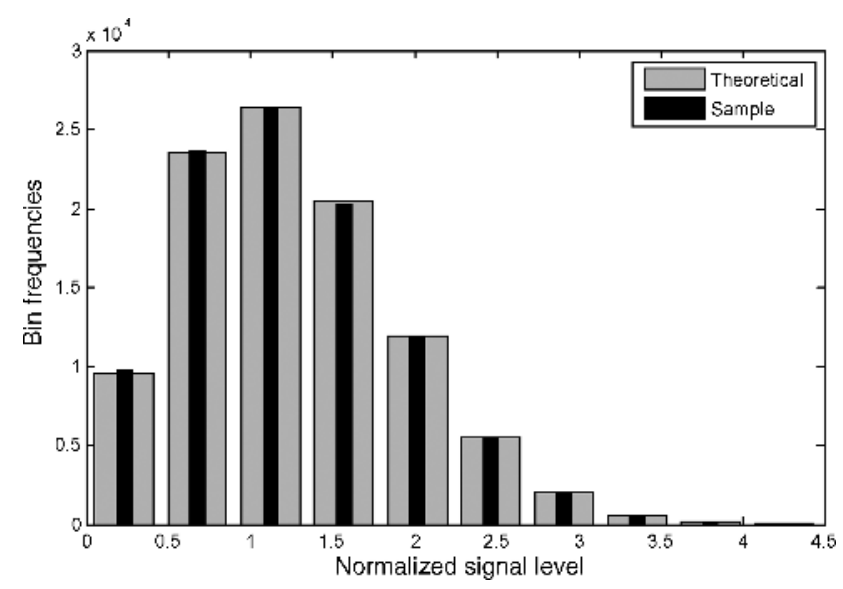

Figure 1.10 Time series and $\sigma=1$ Rayleigh histograms 
threshold which is determined as a function of the so-called significance level of the test. Usually this level is set to $1 \%$ or $5 \%$. Below are the steps to be followed [7] for performing this test:

1. Partition the sample space, $S_{X}$, into the union of $K$ disjoint intervals/bins.

2. Compute the probability, $b_{k}$, that an outcome falls in the $k$-th interval under the assumption that $X$ has the proposed distribution. Thus, $m_{k}=n b_{k}$ is the expected number of outcomes that fall in the $k$-th interval in $n$ repetitions of the experiment.

3. The chi-square measure, $D^{2}$, is defined as the weighted difference between the observed number of outcomes, $N_{k}$, that fall in the $k$-th interval, and the expected number, $m_{k}$,

$$
D^{2}=\sum_{k=1}^{K} \frac{\left(N_{k}-m_{k}\right)^{2}}{m_{k}}
$$

4. If the fit is good, then $D^{2}$ will be small. The hypothesis will be rejected if $D^{2}$ is too large, that is, if $D^{2} \geq t_{\alpha}$, where $t_{\alpha}$ is the threshold for significance level $\alpha$.

The chi-square test is based on the fact that for large $n$, the random variable $D^{2}$ has a distribution which approximately follows a chi-square with $K-1$ degrees of freedom. Thus, the threshold, $t_{\alpha}$, can be computed by finding the point at which (Figure 1.11) $\operatorname{Prob}\left(X \geq t_{\alpha}\right)=\alpha$, where $X$ is a chi-square random variable with $K-1$ degrees of freedom, dof. The chi-square pdf is given by

$$
f(x)=\frac{x^{(K-2) / 2} e^{-x / 2}}{2^{K / 2} \Gamma(k / 2)} \quad x>0
$$

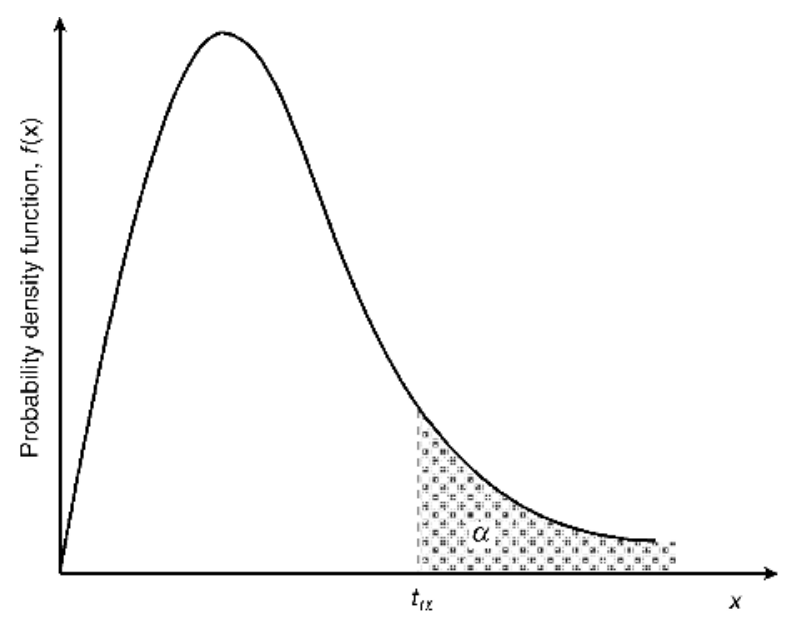

Figure 1.11 Threshold in chi-square test is selected so that $\operatorname{Prob}\left(X \geq t_{\alpha}\right)=\alpha[7]$ 
Table 1.2 Thresholds for significance levels $1 \%$ and 5\%, and different degrees of freedom

\begin{tabular}{rrrrrr}
\hline$K$ & $5 \%$ & $1 \%$ & $K$ & $5 \%$ & $1 \%$ \\
\hline 1 & 3.84 & 6.63 & 12 & 21.03 & 26.22 \\
2 & 5.99 & 9.21 & 13 & 22.36 & 27.69 \\
3 & 7.81 & 11.35 & 14 & 23.69 & 29.14 \\
4 & 9.49 & 13.28 & 15 & 25.00 & 30.58 \\
5 & 11.07 & 15.09 & 16 & 26.30 & 32.00 \\
6 & 12.59 & 16.81 & 17 & 27.59 & 33.41 \\
7 & 14.07 & 18.48 & 18 & 28.87 & 34.81 \\
8 & 15.51 & 20.09 & 19 & 30.14 & 36.19 \\
9 & 16.92 & 21.67 & 20 & 31.41 & 37.57 \\
10 & 18.31 & 23.21 & 25 & 37.65 & 44.31 \\
11 & 19.68 & 24.76 & 30 & 43.77 & 50.89 \\
\hline
\end{tabular}

where $K$ is a positive integer and $\Gamma$ is the Gamma function. This distribution is a special case of the Gamma distribution,

$$
f_{X}(x)=\frac{\lambda(\lambda x)^{\alpha-1} e^{-\lambda x}}{\Gamma(\alpha)} \quad x>0 \quad \text { and } \quad \alpha>0, \lambda>0
$$

when $\alpha=K / 2, K$ is a positive integer, and $\lambda=1 / 2$.

The thresholds for the $1 \%$ and $5 \%$ levels of significance and different degrees of freedom are given in Table 1.2. The number of dof is $K-1$, that is, the number of intervals or bins minus one. It is recommended that, if $r$ is the number of parameters extracted from the data (e.g., mean, standard deviation, etc.), then $D^{2}$ is better approximated by a chi-square distribution with $K-r-1$ degrees of freedom. Each estimated parameter decreases the degrees of freedom by one.

One further recommendation is on how the bins should be taken, since they may significantly influence the outcome of the test [7]. The selection of the intervals must be made so that they are equally probable. Another recommendation is that the expected number of outcomes in each interval be five or more. This will improve the accuracy of approximating the CDF of $D^{2}$ by a chi-square distribution.

We have performed the test first with the output of MATLAB ${ }^{\circledR}$ function hist which splits the range of observed values into intervals of equal length. In project 11 the number of bins has been set to 10 . The resulting value of $D^{2}$ was 20.0873 . This value has to be compared with that in Table 1.2 for $K=10$ (intervals) $-1-1$ (the mode, parameter obtained from the sample) $=8$. The value for $1 \%$ significance is 20.09 which barely exceeds the obtained value for $D^{2}$. In this case the test is passed. For $5 \%$ significance the threshold on the table is 15.51 , meaning that the test is not passed.

As indicated above, it is convenient to use equally probable intervals. Thus, the test was carried out a second time with the intervals and frequencies given in Table 1.3 (BINSequalprobRayleigh). The final value of parameter $D^{2}$ is 15.9884 , in this case the test for $5 \%$ significance is improved and the test is almost passed. 
Table 1.3 Intervals and partial results of chi-square test with equal probability intervals

\begin{tabular}{lcccc}
\hline Bin Min. & Bin Max. & $\begin{array}{c}\text { Measured } \\
\text { frequency }\end{array}$ & $\begin{array}{c}\text { Theoretical } \\
\text { frequency }\end{array}$ & $\begin{array}{c}\text { Elements } \\
\text { of } D^{2}\end{array}$ \\
\hline 0 & 0.4590 & 10159 & 10000 & 2.5281 \\
0.4590 & 0.6680 & 10179 & 10000 & 3.2041 \\
0.6680 & 0.8446 & 10002 & 10000 & 0.0004 \\
0.8446 & 1.0108 & 9782 & 10000 & 4.7524 \\
1.0108 & 1.1774 & 9920 & 10000 & 0.6400 \\
1.1774 & 1.3537 & 10152 & 10000 & 2.3104 \\
1.3537 & 1.5518 & 9857 & 10000 & 2.0449 \\
1.5518 & 1.7941 & 9970 & 10000 & 0.0900 \\
1.7941 & 2.1460 & 9945 & 10000 & 0.3025 \\
2.1460 & $\infty$ & 10034 & 10000 & 0.1156 \\
\hline
\end{tabular}

If the MATLAB ${ }^{\circledR}$ version, including toolboxes, available to the reader contains function gammainc, the significance level can be computed by typing alpha $=1$-gammainc $\left(0.5^{\star} \operatorname{chi}, 0.5^{\star} \mathrm{df}\right)$, where chi2 is $D^{2}$ and $\mathrm{df}$ are the degrees of freedom.

\section{Project 1.2: Shadowing Plus Multipath}

For this project, file series 12 . mat is supplied. This series corresponds to a longer stretch of received signal where both shadowing and multipath effects are present. What we need to do is separate both variations in order to perform an independent study of the shadowing and the multipath-induced variations. Script project 12 is used in this analysis.

A section of the series in file series 12 . mat is shown in Figure 1.12. It corresponds to a signal at $2 \mathrm{GHz}$. File series 12 . mat contains a two-column matrix where the first column represents the traveled distance in meters and the second, the received signal in $\mathrm{dBm}$. It is clear that both slow and fast variations are present. The separation is performed by means of a running mean filter implemented with a rectangular window that is slid through the series. MATLAB $^{\circledR}$ function Conv (convolution) has been used for this purpose. This process gives unreliable samples at the beginning and end of the filtered series which can be discarded.

The original series is in $\mathrm{dBm}$ and should be converted, as in Project 1.1, into voltage units. A window size of $10 \lambda$ (variable NofWavelengths) has been used for separating the fast

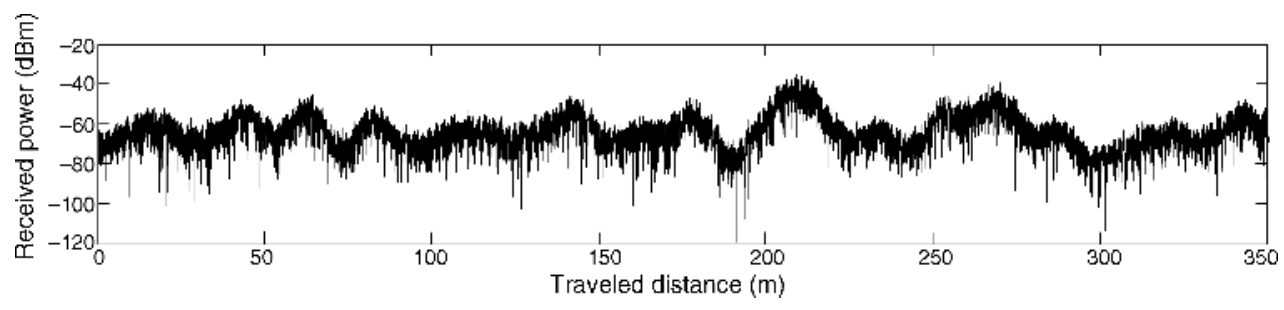

Figure 1.12 Original series 12 time series in $\mathrm{dBm}$ 


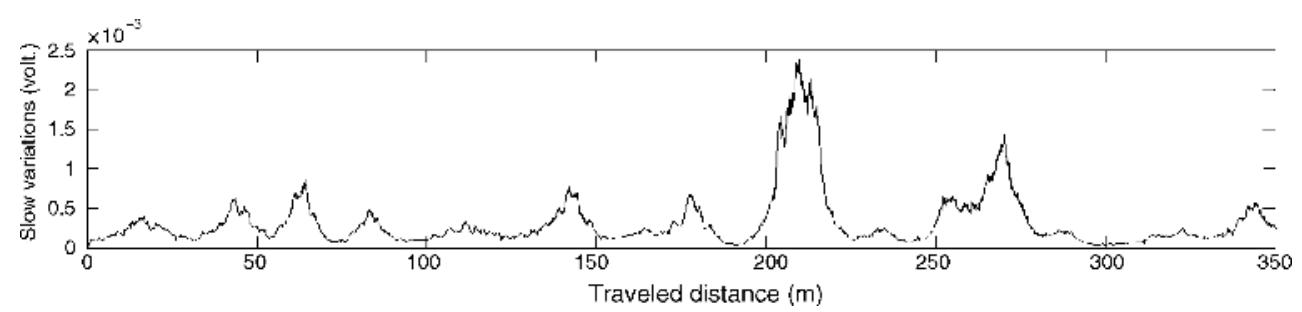

Figure 1.13 Slow variations in volts

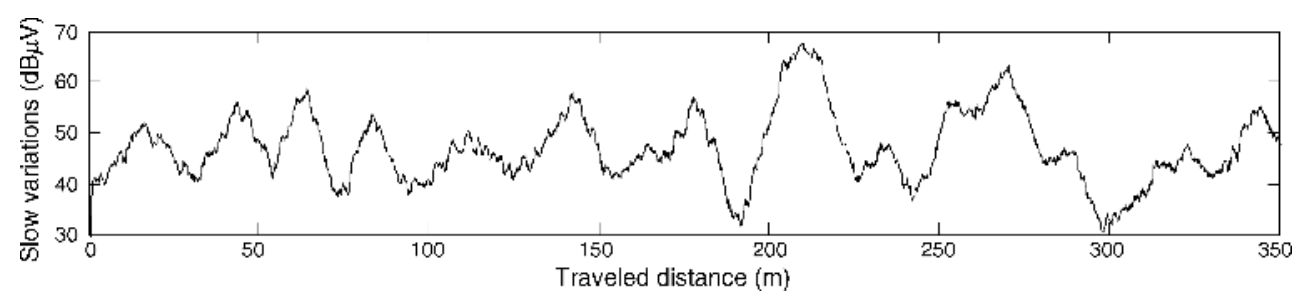

Figure 1.14 Slow variations in $\mathrm{dB} \mu \mathrm{V}$

and slow variations. Other window sizes can be tested. This is left for the reader to try. The sampling spacing is $\lambda / 4$ (variable SamplesperWavelength). Figure 1.13 shows the running mean filtered voltage where the slow variations are clearly visible. High frequency components still remain but this is unavoidable. Figure 1.14 shows the filtered voltage in practical units, $\mathrm{dB} \mu \mathrm{V}, \mathrm{dB}$ relative to $1 \mu \mathrm{V}$, i.e., $V(\mathrm{~dB} \mu \mathrm{V})=20 \log \left[v(\mathrm{Volt}) \times 10^{6}\right]$.

We have first computed the local mean. By dividing the overall voltage by the local mean, we get a normalized voltage with respect to such local mean as shown in Figure 1.15. Figure 1.16 shows both the overall and the slow variations in $\mathrm{dB} \mu \mathrm{V}$. Note the effect of the convolution at the beginning of the plot.

A common assumption in the modeling of the fast variations is that they are Rayleigh distributed as already pointed out in Project 1.1. For the slow variations a lognormal, or normal for the variations in $\mathrm{dB}$, is used. The lognormal distribution will be presented in Chapter 6. Here we remind the reader of some basic facts about the normal or Gaussian

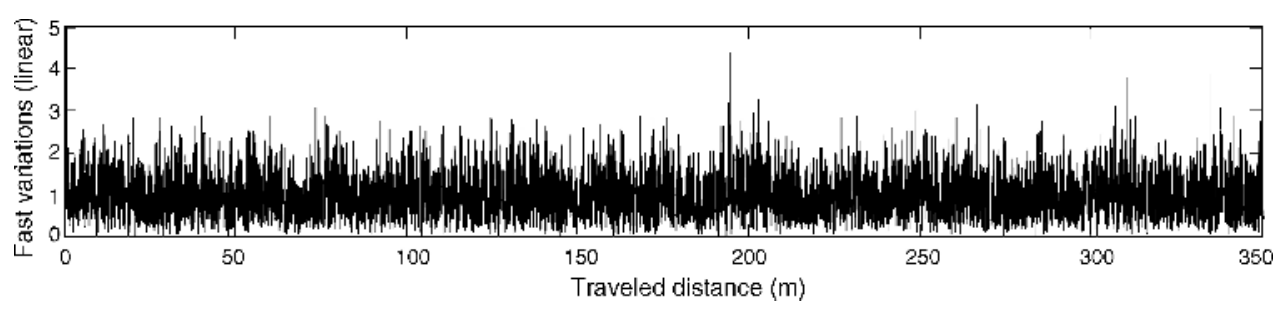

Figure 1.15 Fast variations in linear units 


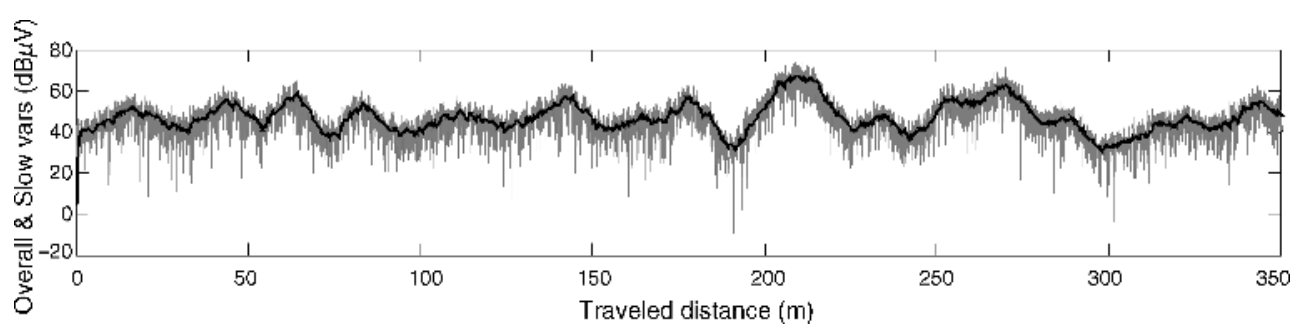

Figure 1.16 Slow and overall variations

distribution that we will need in this project. Its pdf is given by

$$
p(x)=\frac{1}{\sigma \sqrt{2 \pi}} \exp \left[-\frac{1}{2}\left(\frac{x-m}{\sigma}\right)^{2}\right]
$$

and the cumulative distribution is

$$
F(x)=\frac{1}{\sigma \sqrt{2 \pi}} \int_{-\infty}^{x} \exp \left[-\frac{1}{2}\left(\frac{t-m}{\sigma}\right)^{2}\right] \mathrm{d} t=\frac{1}{2}\left[1+\operatorname{erf}\left(\frac{x-m}{\sigma \sqrt{2}}\right)\right]
$$

where erf is the error function.

It is helpful to normalize the random variable $x$ using its mean, $m$, and standard deviation, $\sigma$, i.e., $k=(x-m) / \sigma$, where $k$ is the normalized Gaussian of zero mean and unity standard deviation. Another useful function is

$$
Q(k)=\frac{1}{\sqrt{2 \pi}} \int_{k}^{\infty} \exp \left(-\lambda^{2} / 2\right) d \lambda
$$

Function $Q(k)$ provides an easy way of calculating the probability that random variable $x$ fulfills that $x>m+k \sigma$. This is equivalent to calculating the area under the tail of the pdf (Figure 1.17). Function $Q$ is easily related to the error function or its complementary which

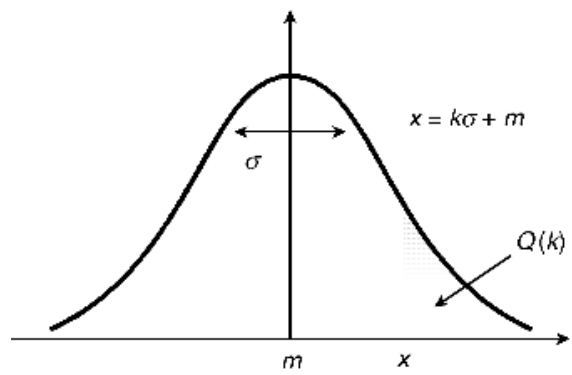

Figure 1.17 Function $Q$, area under the tail of the Gaussian distribution 
Table 1.4 Relevant values of $Q(k)$

\begin{tabular}{cccc}
\hline$k$ & $Q(k)=1-F(k)$ & $k$ & $Q(k)=1-F(k)$ \\
\hline 0 & 0.5 & 1.282 & $10^{-1}$ \\
1 & 0.1587 & 2.326 & $10^{-2}$ \\
2 & 0.02275 & 3.090 & $10^{-3}$ \\
3 & $1.350 \times 10^{-3}$ & 3.719 & $10^{-4}$ \\
4 & $3.167 \times 10^{-5}$ & 4.265 & $10^{-5}$ \\
5 & $2.867 \times 10^{-7}$ & 4.753 & $10^{-6}$ \\
6 & $9.866 \times 10^{-10}$ & 5.199 & $10^{-7}$ \\
& & 5.612 & $10^{-8}$ \\
\hline
\end{tabular}

are available in MATLAB ${ }^{\circledR}$ (functions erf and erfC) through

$$
Q(k)=\frac{1}{2}-\frac{1}{2} \operatorname{erf}\left(\frac{k}{\sqrt{2}}\right)=\frac{1}{2} \operatorname{erfc}\left(\frac{k}{\sqrt{2}}\right)
$$

Table 1.4 provides some practical values of the normalized Gaussian complementary CDF $(1-F)$.

Coming back to our normalized, fast variations series, its mean is 1.0731 (see MATLAB $^{\circledR}$ workspace) which is very close to the expected unitary mean. We will try and model the normalized voltage series by means of a Rayleigh distribution of mean 1.0731 which is equivalent to a Rayleigh with a modal value (Table 1.1) $\sigma=$ mean $/ 1.25=0.8585$.

Figure 1.18 shows a comparison between the sample and the theoretical CDFs. Figure 1.19 shows the CDFs of the slow and overall variations and in Figure 1.20 the sample CDF and a theoretical Gaussian CDF for the slow variations using the sample mean and sample standard deviation, $39.4916 \mathrm{~dB}$ and $3.5708 \mathrm{~dB}$ (MATLAB ${ }^{\circledR}$ workspace). Function GaussianCDF was also used in this study. The fittings are quite good as is to be expected since series 12 was produced using scripts from Chapter 6 implementing this lognormalRayleigh model (Suzuki model). In spite of the series provided being simulated, this should

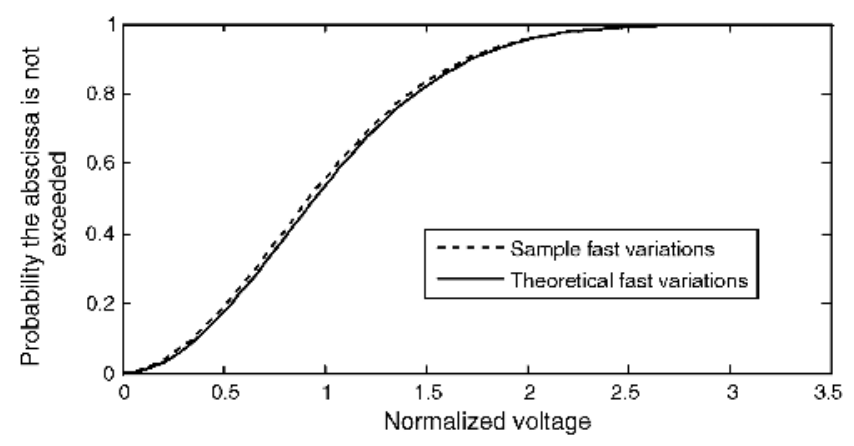

Figure 1.18 Sample and theoretical CDFs for the fast variations 


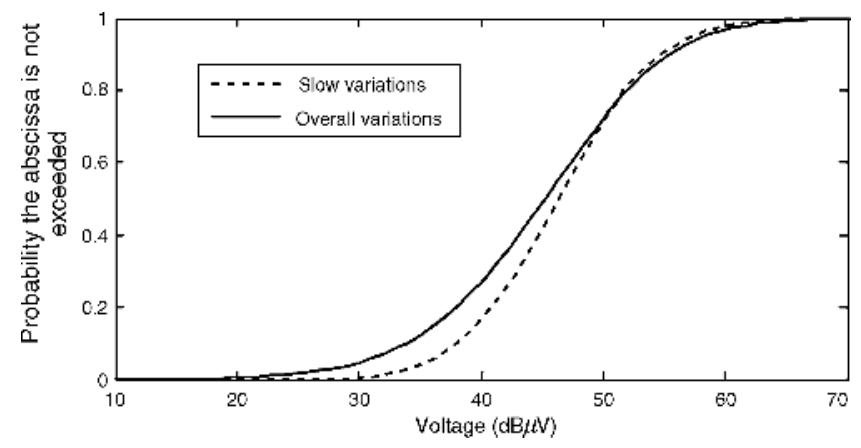

Figure 1.19 Sample CDFs for the slow and overall variations

be a good enough example of how real measurements are processed. Real measurements have been shown to respond quite well to this model [3].

\section{Project 1.3: Complex Envelope Series}

Files series131.mat and series132. mat contain simulated complex time series representing the in-phase, $I$, and quadrature, $Q$, parts of the received signal corresponding to a continuous wave $(\mathrm{CW})$ transmission. The first corresponds to the Rayleigh case and the second to the Rice case. The supplied time series - series131 and series132 - are normalized with respect to the free-space signal level. The two files contain two-column matrices, the first storing the elapsed time, and the second, the complex envelope.

We are interested here in plotting some of the series parameters. For the Rayleigh case, script project131 is used. Figure 1.21 shows the real or in-phase and imaginary or inquadrature components as a function of time. They both should follow, as will be shown in Chapter 4, a Gaussian distribution. Figure 1.21 also shows an in-phase vs. quadrature plot where Lissajous-like curves can be observed which hint at some degree of periodicity in the signals: successive fades and enhancements. Figure 1.22 shows the magnitude of the signal, i.e., $|r|=|I+\mathrm{j} Q|$ computed with MATLAB ${ }^{\circledR}$ function abs (absolute value). This series should

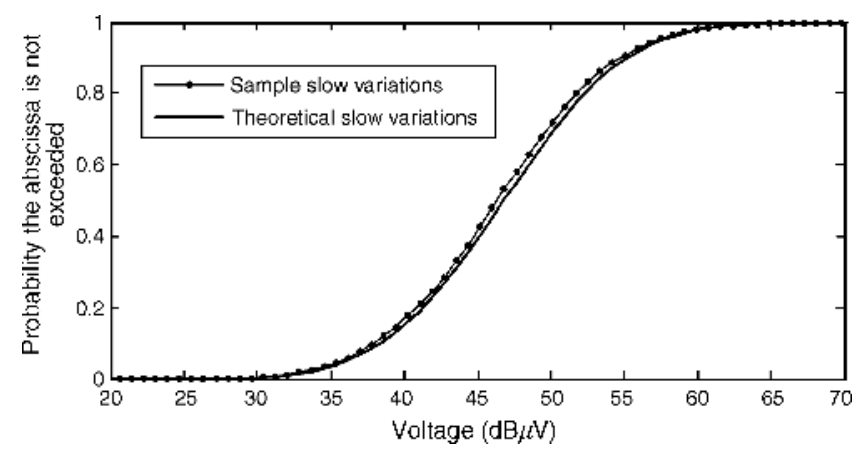

Figure 1.20 Sample and theoretical CDFs for the slow variations 

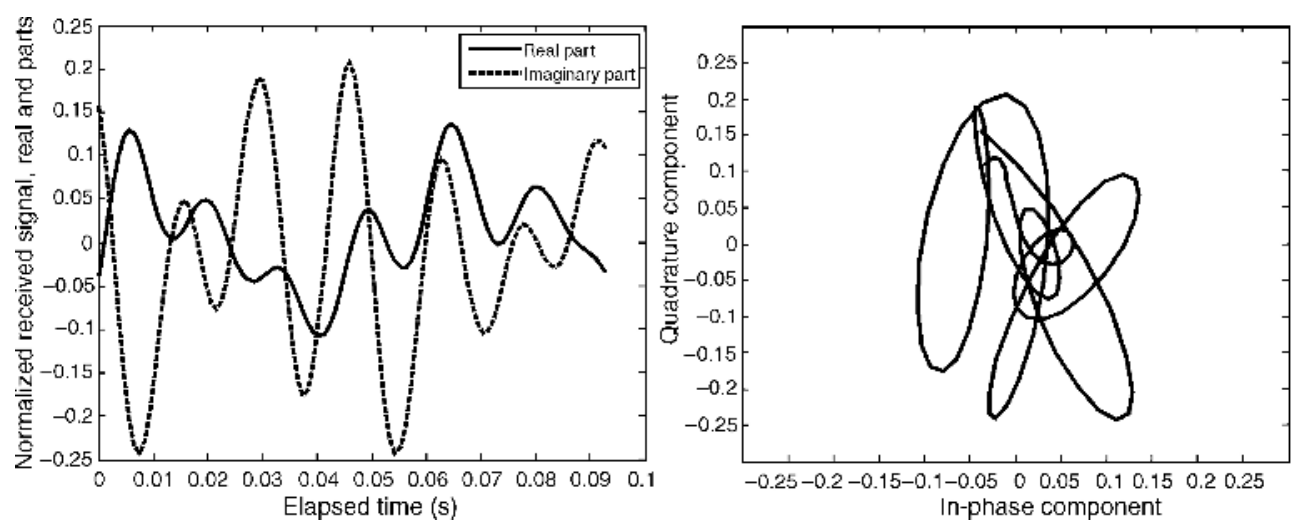

Figure 1.21 Rayleigh series. In-phase and quadrature components, and in-phase vs. quadrature

follow a Rayleigh distribution. Also in the same figure a plot of the magnitude in $\mathrm{dB}$, i.e., $20 \log |I+\mathrm{j} Q|$ is shown. Observe the deep fades and the large overall dynamic range of approximately $30 \mathrm{~dB}$.

Figure 1.23 illustrates the phase (modulo- $\pi$ ) vs. time. This is obtained using MATLAB ${ }^{\circledR}$ function angle. The figure also shows the absolute phase variations obtained using MATLAB ${ }^{\circledR}$ function unwrap after angle. It is clear that the variations are totally random, i.e., neither increasing nor decreasing. The phase variations in this case are usually modeled with a uniform distribution.

For the Rice case, script project132 is used. Figure 1.24 shows the in-phase and quadrature components as a function of time. Again, they both should follow a Gaussian distribution. This figure also shows an $I$ vs. $Q$ plot. Now the Lissajous-like circles are traced about an in-phase level of one (direct signal). Figure 1.25 shows the magnitude of the signal computed with MATLAB ${ }^{\circledR}$ function abs (absolute value). This series should follow a Rice distribution which will be discussed later in Chapter 5. The figure also plots the magnitude in $\mathrm{dB}$. Observe that now, the fades are not so deep, the dynamic range is much smaller and the
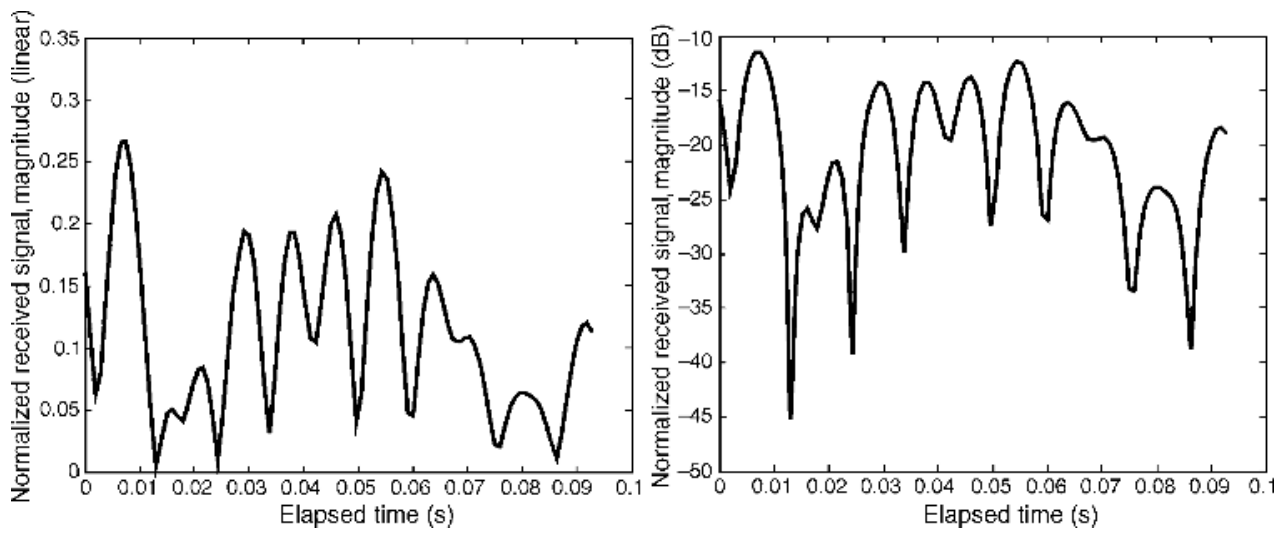

Figure 1.22 Rayleigh series. Magnitude in linear units and dB 

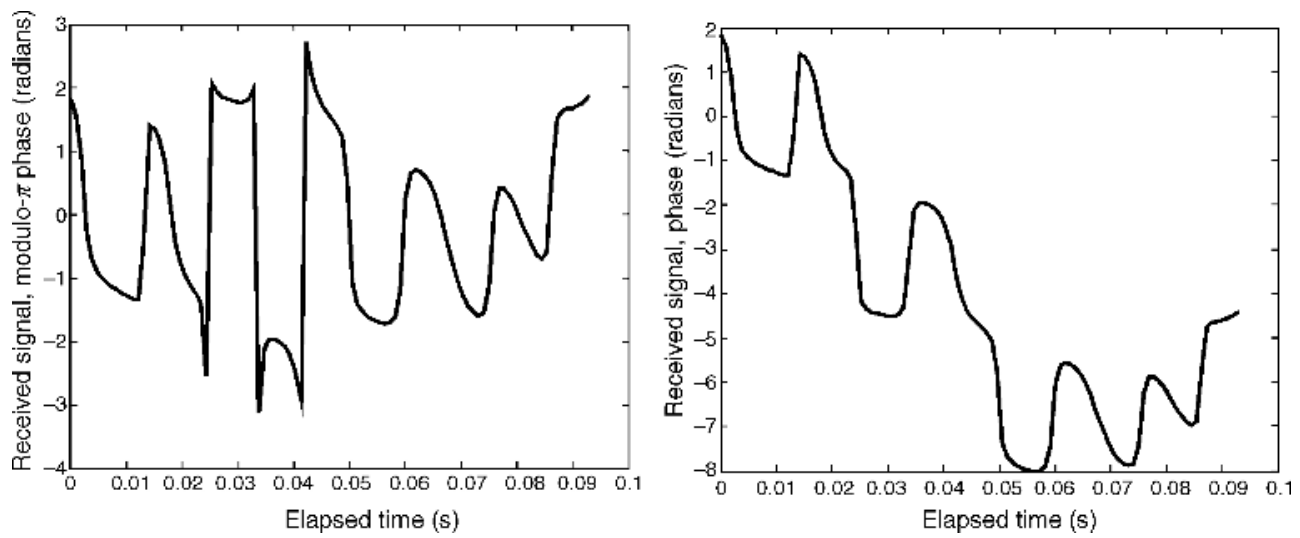

Figure 1.23 Rayleigh series. Modulo- $\pi$ phase variations and absolute phase variations
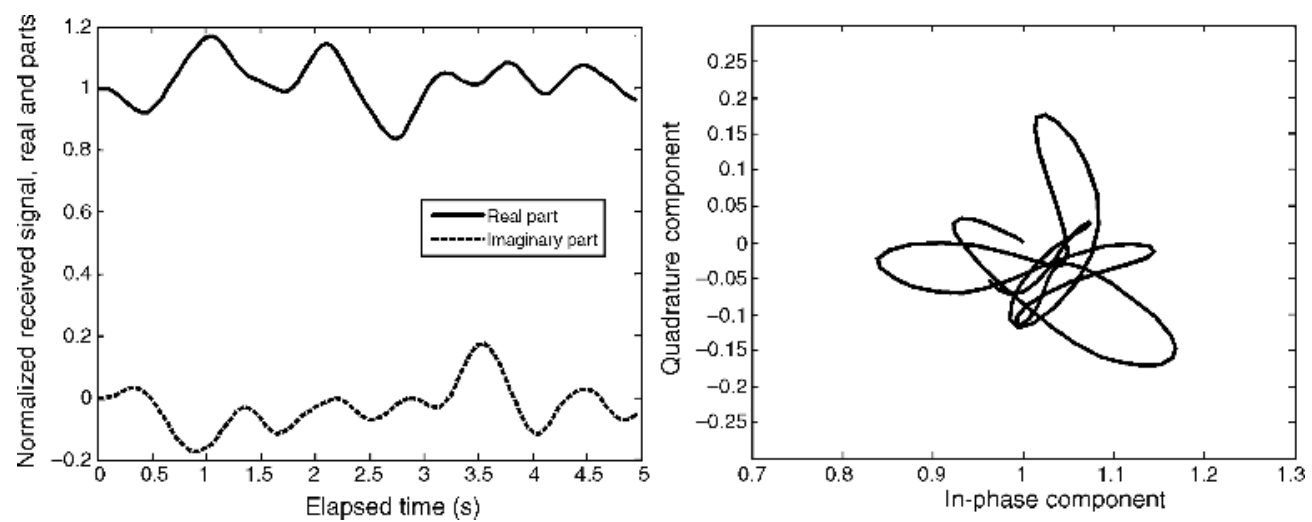

Figure 1.24 Rice series. In-phase and quadrature components, and in-phase vs. quadrature
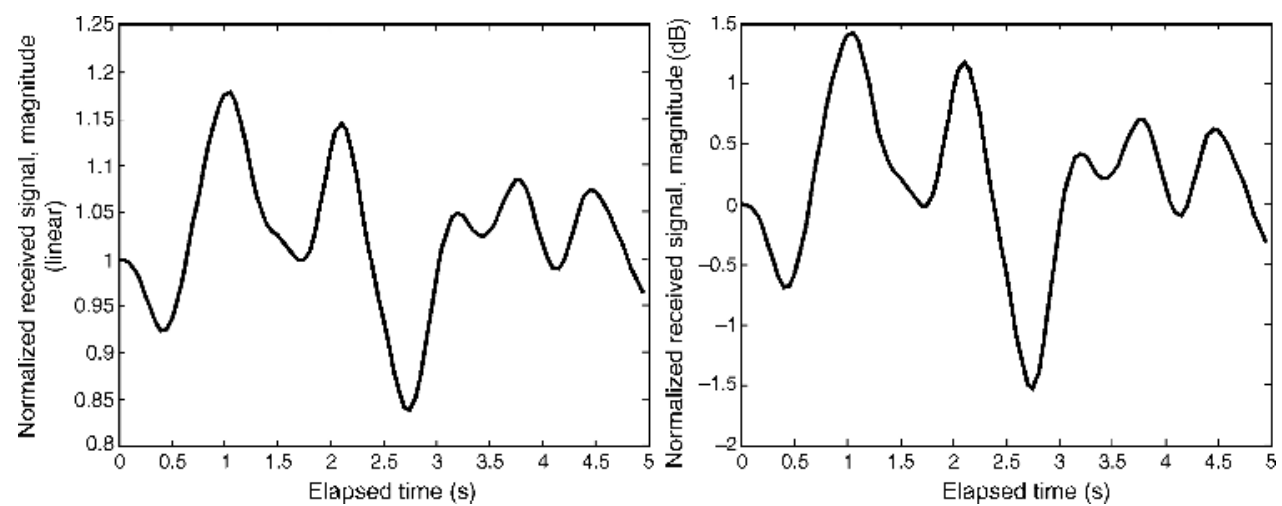

Figure 1.25 Rice series. Magnitude in linear units and in $\mathrm{dB}$ 

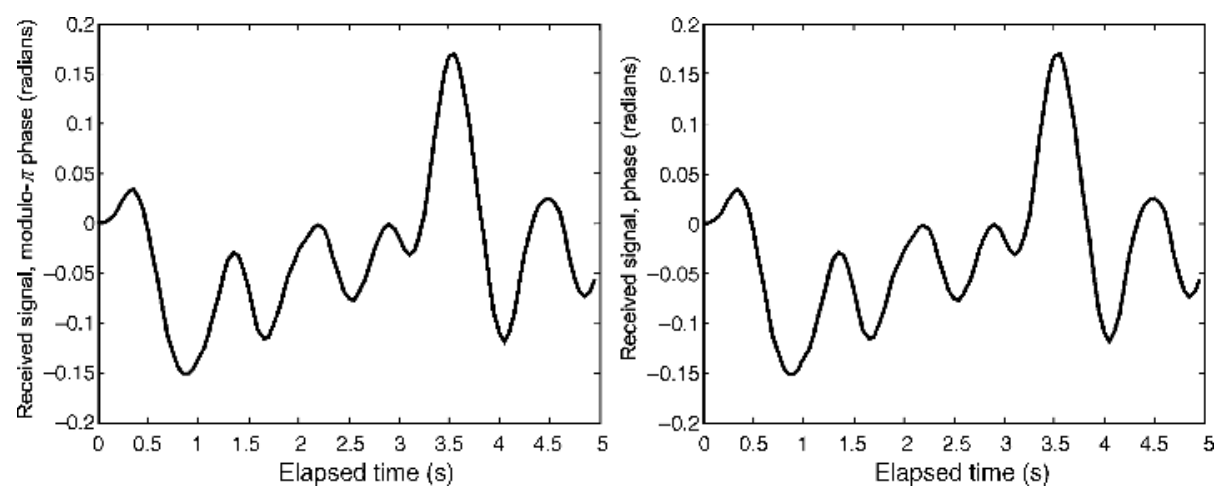

Figure 1.26 Rice series. Modulo- $\pi$ phase variations and absolute phase variations

average level is higher, this due to the direct signal being much stronger than the multipath. Remember that, in the series, the normalized value of one $(0 \mathrm{~dB})$ represents the free-space level.

Figure 1.26 illustrates the phase (modulo- $\pi$ ) vs. time and the absolute phase variations. Unlike in the Rayleigh case, where the variations were totally random, here a clear trend, in this case about zero degrees, is observed in both plots. This clearly shows how the direct signal dominates over the multipath. We leave a more in-detail simulation and analysis of these phenomena for Chapters 4 and 5.

\subsection{Summary}

In this chapter, we have presented a brief introduction to the basic concepts and mechanisms driving the wireless propagation channel. Then, we presented very simple time-series analysis techniques which cover the basics previously introduced, these include the fast variations due to multipath and the combined effects of shadowing and multipath. Finally, we have looked into the complex envelope where we plotted its magnitude and phase. We have also presented the Rayleigh and Rice cases which correspond to harsh and benign propagation conditions. We will now go on to learn more about the various phenomena presented through simulation. This will allow us to become acquainted with a number of fairly complicated concepts in an intuitive way without the need to resort to involved mathematics. This will be done, as in this chapter, in a step-by-step fashion whereby new concepts will be presented as we progress in this familiarization process with the wireless propagation channel.

\section{References}

[1] L.W. Couch. Complex envelope representations for modulated signals (Chapter 1). In Suthan S. Suthersan (Ed.), Mobile Communications Handbook. CRC Press, 1999.

[2] J.M. Hernando \& F. Pérez-Fontán. An Introduction to Mobile Communications Engineering. Artech House, 1999.

[3] W.C.Y. Lee. Mobile Communications Design Fundamentals. Wiley Series in Telecommunications and Signal Processing. John Wiley \& Sons, Ltd, Chichester, UK, 1993. 
[4] M. Hata. Empirical formula for propagation loss in land mobile radio services. IEEE Trans. Veh. Tech., 29(3), 1980, 317-325.

[5] Radiowave Propagation Effects on Next-Generation Fixed-Service Terrestrial Telecommunications Systems. Commission of European Communities. COST 235 Final Report, 1996.

[6] R. Edwards \& J. Durkin. Computer prediction of service areas for VHF mobile radio network. Proc. IEE, 116, 1969, 1493-1500.

[7] A. Leon-Garcia. Probability and Random Processes for Electrical Engineering, Second Edition (International Edition). Addison-Wesley, 1994.

\section{Software Supplied}

In this section, we provide a list of functions and scripts, developed in MATLAB ${ }^{\circledR}$, implementing the various projects and theoretical introductions mentioned in this chapter. They are the following:

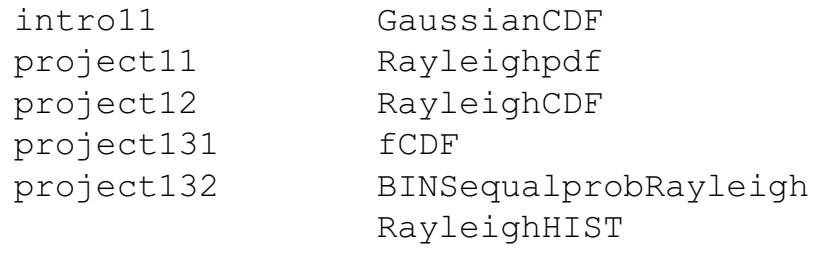

Additionally, the following time series are supplied:

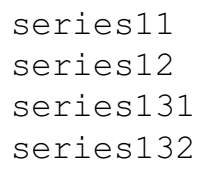


